\title{
The effect of Sep15 gene silencing on the expression of crystall oid protein in human lens epithelial cells
}

\author{
Meng Wang \\ WangMeng people's Hospital of Zoucheng City, Shandong Province Shandong 273500
}

\begin{abstract}
To investigate the effect of Sep15 gene silencing on lens protein expression in human lens epithelial cells. Method: Human lens epithelial cell (HLEC) SRA01/04 was used as the study object, Using MTT method, RT-PCRs, Small molecule RNA interference technology and protein immunoblotting method, The survival rate of hLE cells stimulated by different concentrations of bFGF and Tm (tunicamycin, an endoplasmic reticulum stress inducing reagent), the changes of the mRNA and protein expression levels of intracellular beta-crystallin and GRP78 proteins, and the changes of Sep15 protein expression levels in hLE cells were determined, respectively, to evaluate the effect of Sep15 gene silencing on the expression of lens protein in human lens epithelial cells. Result: First, The survival rate of hLE cells induced by endoplasmic reticulum stress inducing reagents tunicamycin (Tm) and bFGF was investigated by MTT method. The results showed that a certain concentration of Tm and bFGF could inhibit or lead to the death of hLE cells; Secondly, The effects of Tm on the levels of mRNA and protein expression of the endoplasmic reticulum stress marker protein GRP78 and the effects of bFGF on the levels of alpha-, were detected by real-time fluorescence quantitative PCR and protein immunoblotting, respectively, Effects of beta-crystallin mRNA and protein expression levels, To determine the optimal concentration and time of Tm and bFGF on hLE cells, The results showed that $40 \mathrm{ng} / \mathrm{mL}$ of bFGF-treated cells for $48 \mathrm{~h}$ was the optimal reaction condition for bFGF to stimulate the differentiation of lens epithelial cells. Third, The co-action of Tm and bFGF on hLE cells was detected by real-time fluorescence quantitative PCR and protein immunoblotting. For Intracellular GRP78, $\alpha-, \beta$ - crystallin, Effects of mRNA and protein expression levels, The results showed that the occurrence of ER stress could upregulate its expression. Fourth, The changes of Sep15 protein expression level in hLE cells after Sep15 gene silencing and the effects of Tm and bFGF on Sep15 gene silencing in cells were detected by Western blotting. The results showed that the addition of Tm and bFGF basically did not affect the silencing effect of Sep15 gene. Fifth, The expression of GRP78 and $\beta-$ crystallin in hLE cells after silencing of Sep15 gene was detected by Western blot. The results showed that Sep15 may be involved in the process of protecting the differentiation of lens epithelial cells. Conclusion: Sep15 gene silencing has an inhibitory effect on lens protein expression in human lens epithelial cells, and strengthening its clinical research is of positive significance for improving the clinical treatment and prevention of cataract diseases.
\end{abstract}

\section{1 data and methods}

\subsection{Reagents and Instruments for Experiments}

Experimental reagent: DMEM High-sugar medium (Produced by GIBCO) Newborn calf serum and double antibody(Manufactured by hyclone); Trypsin, N-2-hydroxyethylpiperazine-N'-2-ethanesulfonic acid, tetramethylethylenediamine, dithiothreitol, glycerol, Tween, Tris-HCL, Tris-Base, acrylamide, glycine, N, N'-bisacrylamide, sodium dodecyl sulfonate, bromophenol blue and bovine serum albumin (Produced by

Amresco)

Brominated-4,5-dimethyl-2-thiazolyl-2,5-diphenylazozol e (thiazolyl blue, MTT), tunicamycin, Folin phenol reagent and ammonium persulfate(Manufactured by Sigma)TRIzol and Lipofectamine2000 (Manufactured by Invitrogen) bFGF (PeproTech, inc, USA); Reverse transcriptase, dNTP mixture, RNA enzyme inhibitor, fluorescent dye SYBRGreen, Oligo (dT) 18 (produced by ThermoFisher Scientific Company, USA); Protease Inhibitor Cocktail protease inhibitor (produced by Calbiochem Company, Germany); Western and IP cell lysate, rabbit polyclonal anti-Grp78 antibody, mouse monoclonal anti-GAPDH antibody (Beyotime, China) Institute of BiotechnologyProduction); NC film, PVDF film and ECL chemiluminescent reagent (Millipore, USA); rabbit polyclonal anti-beta-Crystallin antibody, rabbit polyclonal anti-Sep15 antibody (abcam, UK);HRP-labeled goat anti-rabbit $\operatorname{IgG}(\mathrm{H}+\mathrm{L})$, goat anti-mouse IgG $(\mathrm{H}+\mathrm{L})$ (produced by Pierce Company, 
USA); NaCL, KCL, dimethyl sulfoxide (DMSO), trichloromethane (CCL3), methanol $(\mathrm{CH} 3 \mathrm{OH})$, ethanol $(\mathrm{C} 2 \mathrm{H} 5 \mathrm{OH})$ and other reagents were all domestic analytical purities; human lens epithelial cell line (HLEC) SRA01/04 (provided by Institute of Oncology, Cancer Hospital, Chinese Academy of Medical Sciences, Beijing).

Experimental instruments: $\mathrm{CO} 2$ incubator (produced by SHEL-LAB Company, USA); YJ-875S clean bench (manufactured by Suzhou Purification Equipment Factory); XSZ-D2 inverted biological microscope (manufactured by Chongqing Optical Equipment Factory); UV-1800 spectrophotometer (manufactured by Leiqi Experimental Equipment Co., Ltd.); ultra-low temperature refrigerator (manufactured by NUAIR Company, Japan); Opticon-2 type PCR instrument (manufactured by MJResearch Company, USA);PHS-3C (manufactured by Shanghai Leimagnetic Instrument Factory); AvantiTMJ-30I refrigerated high-speed centrifuge (manufactured by Beckman Company, USA); ordinary centrifuge (manufactured by Changsha Pingfan Instrument Co., Ltd.); glass plate, 24DN glue maker, DYY-6C electrophoresis equipment (manufactured by Beijing No. 61 Instrument Factory); photosensitive film and dark box (manufactured by Kodark Company, USA);Tanon5200Multi

automatic fluorescence/chemiluminescence imaging analysis system (manufactured by Shanghai Tianneng Technology Co., Ltd.).

\subsection{Experimental method}

In this study, human lens epithelial cells (HLEC) SRA01/04 were used to determine the survival rate of hLE cells after stimulation with different concentrations

Table 1 Effects of different concentrations of tunicamycin and bFGF on the survival rate of hLE cells

\begin{tabular}{ccccc}
\hline $\begin{array}{c}\text { hLECell survival } \\
\text { rate }\end{array}$ & Mild inhibition & $\begin{array}{c}\text { Moderate } \\
\text { inhibition }\end{array}$ & $\begin{array}{c}\text { Significant } \\
\text { inhibition }\end{array}$ & Promotion \\
\hline Tunicamycin & $2.5 \mu \mathrm{g} / \mathrm{mL}$ & $5 \mu \mathrm{g} / \mathrm{mL}$ & $10 \mu \mathrm{g} / \mathrm{mL}$ & \\
bFGF & & & $80 \mathrm{ng} / \mathrm{mL}$ & $40 \mathrm{ng} / \mathrm{mL}$
\end{tabular}

(2) The effects of TM on mRNA and protein expression of ER stress marker GRP78 and bFGF on $\alpha$-, $\beta$ - crystallin mRNA and protein expression were detected by real-time fluorescent quantitative PCR and Western blotting, respectively, to determine the optimal concentration and time of TM and bFGF on hle cells. The results showed that the expression level of GRP78 mRNA and protein was significantly increased when treated with $5 \mu \mathrm{g} / \mathrm{ml}$ TM for 24 hours; The expression level of $\beta$ - crystallin mRNA and protein can be

Table 2 Effect of tunicamycin on protein expression of bFGF and Tm (endoplasmic reticulum stress-inducing reagent tunicamycin) by MTT, RT-PCR, small-molecule RNA interference and protein immunoblotting, and to determine the changes in the expression levels of intracellular beta-lens protein and GRP78 protein. The changes of Sep15 protein expression in hLE cells were also measured to evaluate the effect of Sep15 gene silencing on lens protein expression in human lens epithelial cells.

\subsection{Statistical analysis}

Statistical software SPSS22.0 was used for data statistical analysis, and the count data were expressed by percentage (\%) with the help of $\mathrm{x} 2$ test; the measurement data were expressed by $(+\mathrm{s})$ with $\mathrm{t}$ test. Among them, $\mathrm{P}<$ 0.05 represents significant difference and has statistical significance.

\section{Results}

(1) The effects of endoplasmic reticulum stress-inducing reagents tunicamycin (Tm) and bFGF on the viability of hLE cells were investigated by MTT. The experimental results showed that $\mathrm{Tm}$ with low concentration $(2.5 \mathrm{ug} / \mathrm{mL}, 5 \mathrm{ug} / \mathrm{mL})$ had a certain inhibitory effect on cells, and its inhibitory effect was concentration-dependent; Tm with high concentration (10ug/mL) could significantly increase cell death.As a cell growth factor, bFGF at low concentration $(40 \mathrm{ng} / \mathrm{mL})$ can promote cell growth, but there is no significant change; while bFGF at high concentration $(80 \mathrm{ng} / \mathrm{mL})$ has a significant inhibitory effect on cell growth. significantly increased when bFGF of 40ng / $\mathrm{ml}$ acts on the cells for $48 \mathrm{~h}$, while the expression level of $\alpha$ crystallin mRNA has increased, but there is no significant change, which is related to the distribution of $\alpha-\beta$ crystallin in the lens. Based on the fact that $\beta$ - crystallin is a marker protein of cell differentiation, we choose $\beta$ crystallin in this experiment BFGF of 40ng / $\mathrm{ml}$ acted on the cells for $48 \mathrm{~h}$, which was the best reaction condition for bFGF to stimulate the differentiation of lens epithelial cells.
(3) The effects of Tm and bFGF on the expression levels of GRP78, alpha-, beta-crystallin mRNA and protein in hLE cells were examined by real-time fluorescence quantitative PCR and protein 
immunoblotting. The experimental results showed that the addition of $\mathrm{Tm}$ significantly upregulated the expression levels of GRP78 mRNA and protein in cells on the one hand, but significantly inhibited the expression levels of beta-lens protein mRNA and protein on the other hand. Moreover, the addition order of Tm had no significant effect on the expression level of GRP78 protein in cells, but had a certain effect on the expression level of beta-lens protein. The decrease of beta-lens protein in the group with Tm added first was more significant than that in the group with Tm added later. This indicates that the ER stress induced by adding Tm causes certain damage to the cells, leading to the blockage of the differentiation process of the cells when adding bFGF, and then inhibits the expression of beta-crystallin, that is, the ER stress induced by Tm inhibits the differentiation process of lens cells. The expression level of alpha-crystallin mRNA was increased by the addition of $\mathrm{Tm}$, but there was no significant change, and the sequence of bFGF and Tm addition had no significant effect on the expression level of alpha-crystallin mRNA, indicating that alpha-crystallin, as a chaperone protein of the lens, plays an anti-apoptotic role and can resist the apoptosis induced by Tm-induced endoplasmic reticulum stress. The occurrence of ER stress can upregulate its expression.

(4) The changes of Sep15 protein expression level in hLE cells after Sep15 gene silencing and the effects of $\mathrm{Tm}$ and bFGF on Sep15 gene silencing in cells were detected by Western blotting. The experimental results show that the selected gene silencing sequence can successfully silence Sep15 protein, and the addition of $\mathrm{Tm}$ and bFGF will not affect the silencing effect of Sep15 gene.

(5) The expression levels of GRP78 and beta-crystallin in hLE cells after Sep15 gene silencing were detected by Western blotting. The experimental results showed that the expression level of GRP78 protein did not change significantly after Sep15 gene silencing, but the expression level of beta-crystallin was significantly inhibited, which indicated that Sep15 gene silencing had no significant effect on endoplasmic reticulum stress, but aggravated the inhibition of endoplasmic reticulum stress induced by tunicamycin on the expression of related lens protein during lens epithelial cell differentiation. The results suggest that Sep15 may be involved in the process of protecting lens epithelial cell differentiation.

\section{Discuss}

Cataract is a kind of clinical disease with the highest blindness. At present, it shows an increasing trend year by year. If severe cataract is not cured in time, it will cause blindness and seriously affect the quality of life of patients. The normal development of lens depends on the regular differentiation of lens epithelial cells. If the lens epithelial cells have lesions during lens development, it is very easy to develop into cataract. Relevant data show that single excision of Sep15 gene can induce cataract in mice, but the mechanism is not clear at present.
Therefore, this paper explores the effect of Sep15 gene silencing on human lens. Effect of lens protein expression in epithelial cells. In this study, human lens epithelial cell (HLEC) SRA01/04 was used as the research object, and the changes of mRNA and protein expression levels of intracellular beta-lens protein and GRP78 protein after stimulation with different concentrations of bFGF and Tm (endoplasmic reticulum stress inducing reagent tunicamycin) were measured by MTT, RT-PCR, small molecule RNA interference and protein immunoblotting.Sep15 protein expression levels in hLE cells were also measured to evaluate the effect of Sep15 gene silencing on lens protein expression in human lens epithelial cells. The results showed that a certain concentration of Tm and bFGF could inhibit or lead to the death of hLE cells; $40 \mathrm{ng} / \mathrm{mL}$ of bFGF-treated cells for $48 \mathrm{~h}$ was the best reaction condition for bFGF to stimulate the differentiation of lens epithelial cells; the addition of Tm and bFGF basically did not affect the silencing effect of Sep15 gene; Sep15 may be involved in the process of protecting lens epithelial cell differentiation. The conclusion indicates that Sep15 gene silencing has an inhibitory effect on the expression of lens protein in human lens epithelial cells, and strengthening its clinical research is of positive significance for improving the clinical treatment and prevention of cataract diseases.

\section{Reference}

1. Guo Ning. Effect of Sep15 gene silencing and toxic carotene on cadherin eGuo Ning. The effect of Sep15 gene silencing and Dauricine on the expression of cadherin in human lens epithelial cells [D]. Hubei: Huazhong University of science and technology, 2016

2. He Yaya. Effect of Sep15 gene silencing on integrin expression in human lens epithelial cells induced by high glucose [D]. Hubei: Huazhong University of science and technology, 2016

3. Chen Lu. Effect of Sep15 gene silencing on the expression of crystalloid protein in human lens epithelial cells [D]. Hubei: Huazhong University of science and technology, 2015

4. Dai Jie. The intervention effect and mechanism of selenium on galactose cataract [D]. Hubei: Huazhong University of science and technology, 2017

5. Zhang min. study on the effect of sels gene silencing on the expression of Er selenoprotein mRNA and its Miss target effect in HepG2 cells [D]. Hubei: Huazhong University of science and technology, 2013

6. Du Shaoqing. Regulation of selenoprotein $\mathrm{S}$ and selenoprotein $\mathrm{K}$ in endoplasmic reticulum stress [D]. Hubei: Huazhong University of science and technology, 2010 